\title{
Seventh Cranial Nerve Palsy: The First Sign of Multiple Sclerosis in A Young Children
}

\author{
Dipasquale L, Sciacovelli M, Basilone A, Verrotti di Pianella V, Damiano B, Pedico A, Patisso I, \\ Merola T, Cinalski J, Di Florio L, Marseglia A, Rutigliano I*, Germano M, Pettoello Mantovani M \\ and Sacco $M$
}

Pediatric Unit, IRCCS Casa Sollievo della Sofferenza, San Giovanni Rotondo - Residency Program in Pediatrics, University of Foggia Medical School, Italy

*Corresponding author: Rutigliano I, Pediatric Unit, IRCCS Casa Sollievo della Sofferenza, San Giovanni Rotondo - Residency

Program in Pediatrics, University of Foggia Medical School, Italy

\begin{abstract}
ARTICLE INFO
Received: 蔧 September 18, 2020

Published: 幽 September 29, 2020

Citation: Dipasquale L, Sciacovelli M, Basilone A, Verrotti di Pianella V, Damiano B, et al. Seventh Cranial Nerve Palsy: The First Sign of Multiple Sclerosis in A Young Children. Biomed J Sci \& Tech Res 30(5)2020. BJSTR. MS.ID.005009.

ABSTRACT

We describe the case of a 9-year-old male patient who was admitted for seventh cranial nerve palsy occurred after seven days of frontal headache. The emergency cranial CT scan was negative. For the persistence of symptoms and the onset of vomiting and nystagmus we performed encephalic contrast MRI and MR angiography. The MRI findings were consistent with the McDonalds radiological diagnostic criteria for Multiple Sclerosis (MS), confirmed by the oligoclonal bands positivity in cerebrospinal fluid (CSF). In childhood MS is a rare disease and the clinical onset is variable. Seventh cranial nerve palsy is a very rare first clinical sign of MS and the association between MS and seventh nerve palsy have been rarely reported in literature. As evident in our case, seventh cranial nerve palsy, particularly when associated with other neurological signs and symptoms, should not be underestimated by the clinicians.
\end{abstract}

Abbreviations: MS:Multiple Sclerosis; ADEM: Acute Disseminated Encephalomyelitis; CSF: Cerebrospinal Fluid; CNS: Central Nervous System; NMOSD: Neuromyelitis Optic Spectrum Disorders; ABR: Auditory Brainstem Response; VEP: Visual Evoked Potential

\section{Introduction}

Multiple sclerosis (MS) is a chronic autoimmune inflammatory disease of the central nervous system characterized pathologically by demyelination and subsequent axonal degeneration[1,2]. In childhood,MS is a rare disease;overall incidence is less than 1 per 100000 children and overall prevalence is from 0.7 to 26.92 per 100000 children [3]. It mainly involves adolescents, but rarely it can also affect children younger than 10 years old.The clinical onset in children is variable: isolated optic neuritis and isolated brainstem dysfunction are more frequent, but encephalitic signs, such as disorders of consciousness, headache, vomiting, seizures are also observed [4]. Acute disseminated encephalomyelitis (ADEM) is the initial diagnosis in $16 \%$ of children suffering from MS[5]. The 2017 International McDonald multiple sclerosis diagnostic panel proposed modifications to improve diagnostic accuracy, simplify application of the diagnostic criteria, and enhance timeliness of the diagnosis of multiple sclerosis, reducing the misdiagnosis. These diagnostic criteria perform well in identifying children with multiple sclerosis and they are also applied in children younger than 11 years at presentation without clinical features of ADEM [6]. Over the last three decades, few cases on the association between peripheral demyelinating diseases and MS have been reported in literature[7]. One of the rarest correlations is that between MS and seventh nerve palsy [8-10].Peripheral facial palsy is a common disease in children. Viral infections, trauma, hereditary causes, and malignancies are some of the etiological reasons. In approximately half of cases, the etiology is not identified, suggesting a diagnosis of "Bell's palsy" [11,12].We describe the case of a 9-year-old child who was admitted for a recent headache, unilateral facial palsy, nystagmus and vomiting, and only after performing a brain MRI and other investigations the diagnosis of MS was made. 


\section{Case Report}

A 9-year-old male patient was admitted to the Emergency Room for signs of seventh cranial nerve palsy in the last 24 hours, which occurred after seven days of frontal headache. He had never presented neurological symptoms before. After a Neuropsychiatric evaluation, an emergency cranial CT scan was performed, tested negative for bleeding signs and brain masses. Therefore, a brain MRI was programmed. However, for the recurrence of headache associated with vomiting, A. returned to our department. On physical examination, we observed an asymmetric closure of the right eyelid and buccal asymmetry, like peripheral paresis of the right facial nerve. Moreover, there was a slight horizontal nystagmus on lateral gaze. Laboratory tests (blood count, hepato-renal function, coagulation tests, C-reactive protein, erythrocyte sedimentation rate, immunoglobulin) and instrumental investigation (ECG and EEG) were normal. Pharyngeal swab and Herpes Simplex Virus Serology were negative. Fondus oculi showed a slightly raised papilla with well-defined borders (OD>OS). Encephalic contrast MRI and MR angiography detected multiple nodular areas, hyperintense on FLAIR and Fast Spin Echo T2 sequences, located in the bihemispheric white matter. Most of these findings were in the radiate crown, others were detected in the bilateral cerebellar peduncles and in the left side of the bridge; another one was localized near the posterior side of the left lateral ventricle.

The left frontal polar lesion exhibited a subtle enhancement after administration of the contrast medium. Full spinal MRI showed no lesions. Because of the results of the magnetic resonance imaging, a lumbar puncture was performed. The infectious, chemical, and physical analysis of cerebrospinal fluid (CSF) were normal except for the presence of oligoclonal bands. In order to exclude subclinical involvement of other cranial nerves, we performed VEP (Visual Evoked Potential), ABR (Auditory Brainstem Response) and audiometric examination and the results were found to be normal. Moreover, in the suspicion of a demyelinating disease of the Central Nervous System (CNS), we tested for anti aquaporin4 antibodies (anti-AQP4) and anti-myelin oligodendrocyte glycoprotein antibodies (anti-MOG), which were negative. Due to MRI findings and the positivity to oligoclonal bands in cerebrospinal fluid, the diagnosis of MS was made.

\section{Discussion}

MS is a chronic inflammatory autoimmune disease, characterized by recurrent episodes of demyelination and axonal degeneration of CNS disseminated in time and space [1,2]. MS is a rare pediatric disease and it mainly involves adolescents, but in extremely rare case it can also affect children under 10 [13]. The etiopathogenesis is still not clear and the triggers of the autoimmune reaction is actually unknown. Probably environmental and infectious factors contribute, in genetically predisposed subjects, to the development of MS. Disease onset during pubertal and post-pubertal age is clinically similar to the adult one, and is characterized by ataxia, dizziness, sensory deficits. Clinical presentation in younger children is more common with optic neuritis, isolated brain stem syndrome and encephalopathy symptoms like headache, vomiting, seizure and/or altered consciousness $[4,13]$. In our patient, the first clinical sign was the seventh cranial nerve palsy whose association with MS has been rarely described. Fukazawa and colleagues reported facial palsy as first symptom of MS in $4.7 \%$ of 107 patients with MS [8]. Ivankovic and Demarin described a 35-year-old woman with MS initially misdiagnosed with Bell's palsy[9].

Moreover, Saleh and colleagues described an MS onset with seventh cranial nerve palsy in a 21-year-old patient [10]. Therefore, In MS patients, facial palsy may appear at the onset of the disease and it may be misdiagnosed as Bell's palsy.In our case, peripheral facial paralysis was associated with other neurological signs and symptoms, so the clinical picture was suspicious for a more complex diagnosis, rather than a simple Bell's palsy. In concordance with the Bell's palsy guideline development group (GDG) diagnostic imaging should not routinely performed, excepting for the presence of other neurological findings [14]. Therefore, the brain MRI was warranted, and it revealed a fundamental exam for the diagnosis. In our case the diagnosis of MS was made on clinical, laboratory and radiologic findings, according to McDonalds 2017 criteria $[6,15]$ (Table 1).Indeed, our patient met both spatial dissemination criteria, for the evidence of periventricular and subtentorial lesions, and temporal dissemination criteria, due to the simultaneous presence of a Gadolinium enhancing lesion in left polar frontal area and non-Gadolinium enhancing lesions. Moreover, the presence of oligoclonal bands in cerebrospinal fluid is another criteria of dissemination in time.

Table 1: McDonalds 2017 criteria for the diagnosis of MS.

\begin{tabular}{|c|c|}
\hline $\begin{array}{c}\text { Spatial Dissemination (DIS): } \\
\text { DIS is Defined by the } \\
\begin{array}{c}\text { Simultaneous Presence of One } \\
\text { or More T2 Lesions in at least } 2 \\
\text { of theFollowing Areas: }\end{array}\end{array}$ & $\begin{array}{c}\text { Temporal Dissemination (DIT): } \\
\text { Is Defined by at least One of the } \\
\text { Following Criteria: }\end{array}$ \\
\hline a) Periventricular & $\begin{array}{l}\text { a) A new T2 or Gadolinium + } \\
\text { lesion at a follow-up MRI after } \\
\text { baseline, regardless of the time } \\
\text { between the two MRIs }\end{array}$ \\
\hline b) Juxtacortical or cortical & $\begin{array}{l}\text { b) Simultaneous presence of a } \\
\text { Gadolinium lesions and a non- } \\
\text { Gadolinium lesion at any time }\end{array}$ \\
\hline c) Subtentorial & $\begin{array}{l}\text { c) The presence of oligoclonal } \\
\text { bands in the }\end{array}$ \\
\hline d) Spinal cord & \\
\hline
\end{tabular}

Useful investigation for differential diagnoses between CNS demyelinating inflammatory disease is testing for anti-AQP4 and anti-MOG antibodies. Anti-AQP4 antibodies are eleveted in neuromyelitis optic spectrum disorders (NMOSD)[16]. Anti-MOG antibodies are more frequent in ADEM and numerous pediatric studies suggest that positivity for these antibodies is predictive 
of CNS demyelinating disease other than MS [17-19].In our case, negativity for both anti AQP4 antibodies and anti MOG antibodies supported the diagnosis of MS.In conclusion, we can assert that making diagnosis of MS might be complex, given the variability of clinical manifestation at the onset especially in young children. As evident in our case, the seventh cranial nerve palsy, particularly when associated with other neurological signs and symptoms, should not be underestimated by the clinicians. So, it is important to keep in mind that in rare cases, peripheral facial palsy can also be a presenting sign of MS.

\section{References}

1. Calabresi PA (2004) Diagnosis and management of multiple sclerosis. Am Fam Physician 70(10): 1935-1944.

2. Goldenberg MM (2012) Multiple sclerosis review. PT 37(3): 175-184.

3. Jeong A, Oleske DM, Holman J (2019) Epidemiology of Pediatric-Onset Multiple Sclerosis: A Systematic Review of the Literature. J Child Neurol 34(12): 705-712.

4. Renoux C, Vukusic S, Mikaeloff Y, Gilles Edan, Michel Clanet, et al. (2007) Natural history of multiple sclerosis with childhood onset. N Engl J Med 356(25): 2603-2613.

5. Banwell B, Krupp L, Kennedy J, Raymond Tellier, Silvia Tenembaum, et al. (2007) Clinical features and viral serologies in children with multiple sclerosis: A multinational observational study. Lancet Neurol 6(9): 773781.

6. Fadda G, Brown RA, Longoni G, Denise A Castro, Julia O Mahony, et al. (2018) MRI and laboratory features and the performance of international criteria in the diagnosis of multiple sclerosis in children and adolescents: A prospective cohort study. Lancet Child Adolesc Health 2(3): 191-204.

7. Kwon JY, Kim JY, Jeong JH, Park KD (2008) Multiple sclerosis and peripheral multifocal demyelinating neuropathies occurring in a same patient. J Clin Neurol 4: 51-57.

\section{ISSN: 2574-1241}

DOI: 10.26717/BJSTR.2020.30.005009

Rutigliano I. Biomed J Sci \& Tech Res

This work is licensed under Creative Commons Attribution 4.0 License

Submission Link: https://biomedres.us/submit-manuscript.php
8. Fukazawa T, Moriwaka F, Hamada K, Hamada T, Tashiro K (1997) Facial palsy in multiple sclerosis. J Neurol 244(10): 631-633.

9. Ivanković M, Demarin V (2011) From recurrent peripheral facial palsy to multiple sclerosis. Acta Clin Croat 50(3): 419-421.

10. Saleh C, Patsi O, Mataigne F, Beyenburg S (2016) Peripheral (Seventh) Nerve Palsy and Multiple Sclerosis: A Diagnostic Dilemma - A Case Report. Case Rep Neurol 8(1): 27-33.

11. Shargorodsky J, Lin HW, Gopen Q (2010) Facial nerve palsy in the pediatric population. Clin Pediatr (Phila) 49: 411

12. Karalok ZS, Taskin BD, Ozturk Z, Gurkas E, Koc TB, et al. (2018) Childhood peripheral facial palsy. Childs Nerv Syst 34(5): 911-917.

13. Ferreira ML, Machado MI, Dantas MJ, Moreira AJ, Souza AM (2008) Pediatric multiple sclerosis analysis of clinical and epidemiological aspects according to National MS Society Consensus 2007. Arq Neuropsiquiatr 66(3B): 665-670.

14. Baugh RF, Basura GJ, Ishii LE, Seth R Schwartz, Caitlin Murray Drumheller et al. (2013) Clinical practice guideline: Bell's palsy. Otolaryngol Head Neck Surg 149(3 Suppl): S1-S27.

15. Thompson AJ, Banwell BL, Barkhof F, William M Carroll, Timothy Coetzee, et al. (2018) Diagnosis of multiple sclerosis: 2017 revisions of the McDonald criteria. Lancet Neurol 17(2): 162-173.

16. Gombolay GY, Chitnis T (2018) Pediatric Neuromyelitis Optica Spectrum Disorders. Curr Treat Options Neurol 20(6): 19.

17. Hacohen Y, Absoud M, Deiva K, Cheryl Hemingway, Petra Nytrova, et al (2015) Myelin oligodendrocyte glycoprotein antibodies are associated with a non-MS course in children. Neurol Neuroimmunol Neuroinflamm 2(2): e81.

18. Hennes EM, Baumann M, Schanda K, Banu Anlar, Barbara Bajer Kornek, et al. (2017) Prognostic relevance of MOG antibodies in children with an acquired demyelinating syndrome. Neurology 89(9): 900-908.

19. Ketelslegers IA, Van Pelt DE, Bryde S, Rinze F Neuteboom, Coriene E Catsman Berrevoets, et al. (2015) Anti-MOG antibodies plead against MS diagnosis in an Acquired Demyelinating Syndromes cohort. Mult Scler 21(12): 1513-1520.

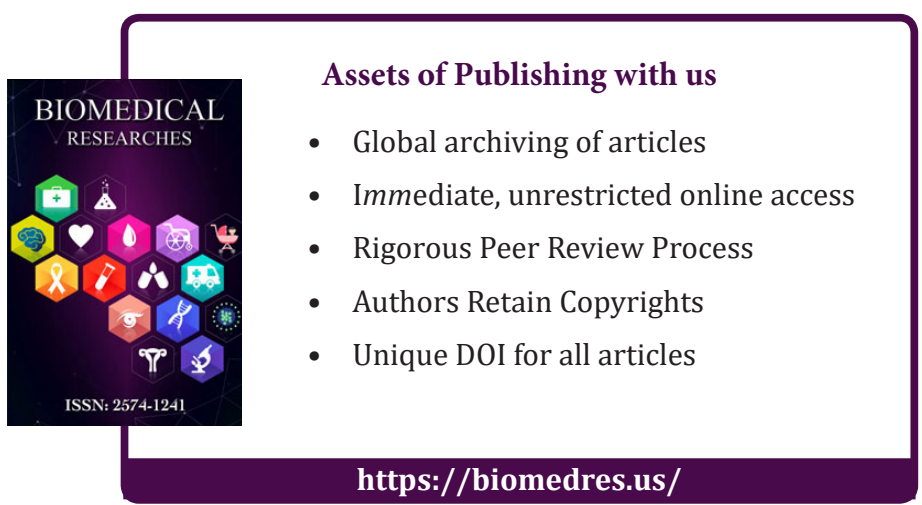

